\title{
Three-loop matching of the vector current
}

\author{
Peter Marquard, ${ }^{1,2}$ Jan H. Piclum, ${ }^{3,4}$ Dirk Seidel, ${ }^{5}$ and Matthias Steinhauser ${ }^{1}$ \\ ${ }^{1}$ Institut für Theoretische Teilchenphysik, Karlsruhe Institute of Technology (KIT), D-76128 Karlsruhe, Germany \\ ${ }^{2}$ Deutsches Elektronen Synchrotron DESY, Platanenallee 6, D-15738 Zeuthen, Germany \\ ${ }^{3}$ Institut für Theoretische Teilchenphysik und Kosmologie, RWTH Aachen, D-52056 Aachen, Germany \\ ${ }^{4}$ Physik-Department T31, Technische Universität München, D-85748 Garching, Germany \\ ${ }^{5}$ Theoretische Physik 1, Universität Siegen, D-57068 Siegen, Germany
}

(Dated: January 15, 2014)

\begin{abstract}
We evaluate the three-loop corrections to the matching coefficient of the vector current between Quantum Chromodynamics (QCD) and non-relativistic QCD. The result is presented in the $\overline{\mathrm{MS}}$ scheme where large perturbative corrections are observed. The implications on the threshold production of top quark pairs are briefly discussed.
\end{abstract}

PACS numbers: 12.38.Bx, 14.65.Ha, 14.65.Fy

\section{INTRODUCTION}

In the recent years effective field theories constructed from Quantum Chromodynamics (QCD) have been enormously successful to describe phenomena where masses and momenta follow certain limits. Among them is nonrelativistic QCD (NRQCD) [1, 2] which is applicable to a system of two heavy quarks moving with small relative velocity. Next to properties of the $\psi$ and $\Upsilon$ families also the threshold production of top quark pairs is among the prominent examples (see, e.g., Ref. [3] for a review).

The common method to construct an effective theory is based on the so-called matching procedure: appropriately chosen Green's functions are computed in the full and effective theory and equality is required up to powersuppressed terms. In this way the couplings of the effective operators (i.e. the matching coefficients) are determined which completely specifies the effective theory.

A crucial operator both in QCD and NRQCD is the vector current of a heavy quark-antiquark pair. The corresponding matching coefficient enters as a building block in a variety of physical observables, for example the bottom-quark mass from $\Upsilon$ sum rules (see, e.g., Refs. [4, 5] for recent analyses) and top-quark threshold production at a future electron positron linear collider [6]. The latter process allows for an extraction of the topquark mass with an accuracy below $100 \mathrm{MeV}$ [7 9] an improvement of about an order of magnitude as compared to the current results from the Fermilab Tevatron or the the CERN Large Hadron Collider [10].

Several quantities are needed in order to perform a third-order analysis of a heavy quark-antiquark system at threshold. Ultrasoft effects have been considered in Refs. [11, 12], the three-loop static potential has been computed in Refs. [13 15] and in Ref. [16, 17] a preliminary analysis of the top-quark threshold production cross section has been presented including also third-order potential effects. Details on the potential contributions can be found in Refs. [18, 19]. In this paper we compute the three-loop matching coefficient between the vector current in QCD and NRQCD. Thus all ingredients are avail- able to obtain the complete next-to-next-to-next-to leading order QCD prediction of the cross section $e^{+} e^{-} \rightarrow t \bar{t}$ close to threshold or the decay width of the $\Upsilon(1 S)$ meson to leptons. The results for the latter are presented in an accompanying paper 20] where all building blocks are combined to a phenomenological analysis.

\section{VECTOR CURRENTS IN QCD AND NRQCD}

The vector current in the full theory (QCD) is given by

$$
j_{v}^{\mu}=\bar{Q} \gamma^{\mu} Q
$$

where $Q$ denotes a generic heavy quark with mass $m_{Q}$. On the other hand in the effective theory (NRQCD) the current is represented by an expansion in $1 / m_{Q}$ where at each order effective operators have to be considered which are multiplied by coefficient functions. The leading contribution involves one operator given by

$$
\tilde{j}^{k}=\phi^{\dagger} \sigma^{k} \chi,
$$

where $\phi$ and $\chi$ are two-component Pauli spinors for quark and anti-quark, respectively, and $\sigma^{k}(k=1,2,3)$ are the Pauli matrices. Hence, the matching coefficient of the vector current is defined through

$$
j_{v}^{k}=c_{v}(\mu) \tilde{j}^{k}+\mathcal{O}\left(\frac{1}{m_{Q}^{2}}\right) .
$$

Note that the 0 -component of $j_{v}^{\mu}$ is only relevant for the power-suppressed contributions.

The purpose of this paper is the evaluation of the purely gluonic three-loop corrections to $c_{v}$. The fermionic contributions have already been considered in Refs. 21, 22].

In order to compute $c_{v}$ it is convenient to consider onshell vertex corrections involving the currents $j_{v}^{k}$ and $\tilde{j}^{k}$. After taking into account the wave function renormalization one obtains (see also Ref. [18])

$$
Z_{2} \Gamma_{v}=c_{v} \tilde{Z}_{2} \tilde{Z}_{v}^{-1} \tilde{\Gamma}_{v}+\ldots,
$$




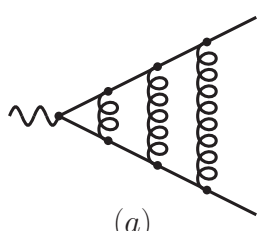

(a)

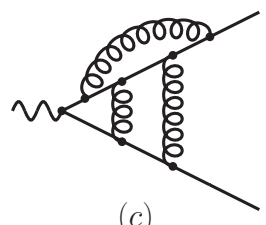

(c)

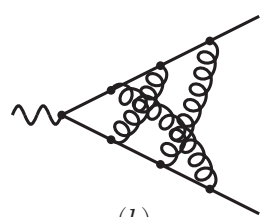

(b)

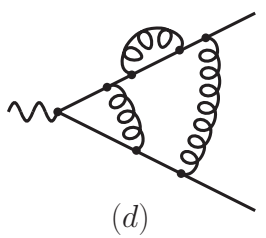

FIG. 1: Feynman diagrams contributing to $\Gamma_{v}$. Straight and curly lines denote heavy quarks with mass $m_{Q}$ and gluons, respectively.

where the quantities with a tilde are defined in the effective theory and the ellipsis represents terms suppressed by the heavy-quark mass. $\tilde{Z}_{v}^{-1}$ is the renormalization constant of the current $\tilde{j}^{k}$ which is used to subtract the remaining poles after renormalization. These poles are due to the separation of long and short distance contributions in the effective theory. In order to evaluate physical quantities it is important that the same subtraction scheme is also adopted in the contributions originating from the effective theory [11, 12]. It is well-known that in the full theory the renormalization constant of the vector current is equal to one.

In Eq. (4) $Z_{2}$ is the on-shell wave function renormalization constant which has been computed up to three-loop accuracy in Refs. 23 25]. $\Gamma_{v}$ denotes the one-particle irreducible vertex diagrams with on-shell quarks carrying momenta $q_{1}$ and $q_{2}$. It incorporates all one-particle irreducible vertex graphs and the corresponding counterterms for $m_{Q}$ and $\alpha_{s}$. Sample Feynman diagrams are shown in Fig. 1.

The counterparts of $\Gamma_{v}$ and $Z_{2}$ in the effective theory can be found on the right-hand side of Eq. (4). It is convenient to apply the threshold expansion [26, 27] to Eq. (4). This requires the identification of the hard, soft, potential and ultrasoft momentum regions in the integrals contributing to $\Gamma_{v}$ and $\tilde{\Gamma}_{v}$. Since NRQCD is obtained from QCD by integrating out the hard modes one has by construction that the soft, potential and ultrasoft modes agree in $\Gamma_{v}$ and $\tilde{\Gamma}_{v}$ and thus drop out from Eq. (4). As a consequence $\Gamma_{v}$ is evaluated for $q^{2}=\left(q_{1}+q_{2}\right)^{2}=4 m_{Q}^{2}$, which corresponds to the leading term of the hard integration region, and $\tilde{\Gamma}_{v}=1$. Furthermore, we have $\tilde{Z}_{2}=1$.

There are several technical difficulties which one has to overcome in order to compute the vertex corrections. Among them are the large number of diagrams which leads to several thousand Feynman integrals to be evaluated in the first place, their reduction to a small set of about 100 basis integrals, so-called master integrals, and the evaluation of the latter in an expansion in
$\epsilon=(4-D) / 2$, where $D$ is the space-time dimension. The last two tasks become more complicated by the additional condition $q^{2}=4 m_{Q}^{2}$ on the external momentum. An automated setup for the calculation has been described in Ref. 22] and applied to the fermionic contributions. Its core parts are a powerful implementation of Laporta's algorithm in the program CRUSHER [28], and FIESTA [29] 31] which is based on sector decomposition and is used for the numerical integration of the master integrals. The main differences to the gluonic part considered in this paper are the larger number of diagrams and the increased complexity of the integrals which have to be reduced to master integrals. Furthermore, the master integrals are more numerous and more involved.

\section{MATCHING COEFFICIENT TO ORDER $\alpha_{s}^{3}$}

Before discussing the matching coefficient it is instructive to consider the renormalization constant $\tilde{Z}_{v}$. The analytical results can be extracted from Refs. 11, 21, 32, 33].

$$
\begin{aligned}
\tilde{Z}_{v} & =\left(\frac{\alpha_{s}^{\left(n_{l}\right)}(\mu)}{\pi}\right)^{2} \frac{C_{F} \pi^{2}}{\epsilon}\left(\frac{1}{12} C_{F}+\frac{1}{8} C_{A}\right) \\
& +\left(\frac{\alpha_{s}^{\left(n_{l}\right)}(\mu)}{\pi}\right)^{3} C_{F} \pi^{2} \\
& \times\left\{C_{F}^{2}\left[\frac{5}{144 \epsilon^{2}}+\left(\frac{43}{144}-\frac{1}{2} \ln 2+\frac{5}{48} L_{\mu}\right) \frac{1}{\epsilon}\right]\right. \\
& +C_{F} C_{A}\left[\frac{1}{864 \epsilon^{2}}+\left(\frac{113}{324}+\frac{1}{4} \ln 2+\frac{5}{32} L_{\mu}\right) \frac{1}{\epsilon}\right] \\
& +C_{A}^{2}\left[-\frac{1}{16 \epsilon^{2}}+\left(\frac{2}{27}+\frac{1}{4} \ln 2+\frac{1}{24} L_{\mu}\right) \frac{1}{\epsilon}\right] \\
& +T n_{l}\left[C_{F}\left(\frac{1}{54 \epsilon^{2}}-\frac{25}{324 \epsilon}\right)+C_{A}\left(\frac{1}{36 \epsilon^{2}}-\frac{37}{432 \epsilon}\right)\right] \\
& \left.+C_{F} T n_{h} \frac{1}{60 \epsilon}\right\}+\mathcal{O}\left(\alpha_{s}^{4}\right),
\end{aligned}
$$

where $C_{A}=N_{c}, C_{F}=\left(N_{c}^{2}-1\right) /\left(2 N_{c}\right)$ and $T=1 / 2$ for a $\mathrm{SU}\left(N_{c}\right)$ gauge group and $L_{\mu}=\ln \left(\mu^{2} / m_{Q}^{2}\right)$. Note that the strong coupling is defined in the effective theory with $n_{l}$ active quarks where $n_{l}+n_{h}$ is the total number of quark flavors. In our case we have $n_{h}=1$, however, we keep $n_{h}$ in the formulae for convenience.

From our calculation we can extract the renormalization constant $\tilde{Z}_{v}$ and compare with Eq. (5). The central values of our numerical coefficients agree at the per cent level with the analytical result of Eq. (5) which constitutes a first non-trivial check and provides quite some confidence in the overall set-up of our calculation.

We write the perturbative expansion of the matching 
coefficient in the form

$$
\begin{aligned}
c_{v}=1 & +\frac{\alpha_{s}^{\left(n_{l}\right)}(\mu)}{\pi} c_{v}^{(1)}+\left(\frac{\alpha_{s}^{\left(n_{l}\right)}(\mu)}{\pi}\right)^{2} c_{v}^{(2)} \\
& +\left(\frac{\alpha_{s}^{\left(n_{l}\right)}(\mu)}{\pi}\right)^{3} c_{v}^{(3)}+\mathcal{O}\left(\alpha_{s}^{4}\right),
\end{aligned}
$$

and decompose $c_{v}^{(3)}$ according to the color structures as

$$
\begin{aligned}
& c_{v}^{(3)}= \\
& \quad C_{F}\left[C_{F}^{2} c_{F F F}+C_{F} C_{A} c_{F F A}+C_{A}^{2} c_{F A A}\right. \\
& \quad+T n_{l}\left(C_{F} c_{F F L}+C_{A} c_{F A L}+T n_{h} c_{F H L}+T n_{l} c_{F L L}\right) \\
& \left.\quad+T n_{h}\left(C_{F} c_{F F H}+C_{A} c_{F A H}+T n_{h} c_{F H H}\right)\right] \\
& \quad+\text { singlet terms. }
\end{aligned}
$$

Note that all color factors of the non-singlet part can be expressed in terms of $C_{F}, C_{A}$, and $T$. In this paper we do not consider the singlet contribution where the external current does not couple to the fermion line in the final state. At two-loop order such contributions have been computed [43] for axial-vector, scalar and pseudo-scalar currents in Ref. [34]. Their numerical effect in those cases is below $3 \%$ as compared to the non-singlet contributions and thus quite small.

Whereas the one- 35] and two-loop [32, 34, 36] terms have been known since long the fermionic three-loop corrections became available only a few years ago 21, 22. The so-called renormalon contribution, consisting of the one-loop diagram with arbitrary many massless quark loop insertions in the gluon propagator, has been computed in Ref. [37]. Supersymmetric one-loop corrections to $c_{v}$ have been computed in Ref. [38].

In the following we present the results for the individual coefficients in Eq. (77) parameterized in terms of $\alpha_{s}^{\left(n_{l}\right)}\left(m_{Q}\right)$. The reconstruction of the full dependence on the renormalization scale is straightforward; the corresponding expressions can be obtained from [39]. Our results read [4]

$$
\begin{aligned}
c_{v}^{(1)}= & -2 C_{F}, \\
c_{v}^{(2)}= & \left(-\frac{151}{72}+\frac{89}{144} \pi^{2}-\frac{5}{6} \pi^{2} \ln 2-\frac{13}{4} \zeta(3)\right) C_{A} C_{F} \\
& +\left(\frac{23}{8}-\frac{79}{36} \pi^{2}+\pi^{2} \ln 2-\frac{1}{2} \zeta(3)\right) C_{F}^{2} \\
& +\left(\frac{22}{9}-\frac{2}{9} \pi^{2}\right) C_{F} T n_{h}+\frac{11}{18} C_{F} T n_{l} \\
& -\frac{1}{2} \pi^{2}\left(\frac{1}{2} C_{A}+\frac{1}{3} C_{F}\right) C_{F} L_{\mu}, \\
c_{F F F}= & 36.55(0.53) \\
& +\left(-\frac{9}{16}+\frac{3}{2} \ln 2\right) \pi^{2} L_{\mu}-\frac{5}{32} \pi^{2} L_{\mu}^{2}, \\
c_{F F A}= & -188.10(0.83) \\
& +\left(-\frac{59}{108}-\frac{3}{4} \ln 2\right) \pi^{2} L_{\mu}-\frac{47}{576} \pi^{2} L_{\mu}^{2},
\end{aligned}
$$

$$
\begin{aligned}
c_{F A A}= & -97.81(0.38) \\
& +\left(-\frac{2}{9}-\frac{3}{4} \ln 2\right) \pi^{2} L_{\mu}+\frac{1}{6} \pi^{2} L_{\mu}^{2}, \\
c_{F F L}= & 46.691(0.006)+\frac{25}{108} \pi^{2} L_{\mu}-\frac{1}{18} \pi^{2} L_{\mu}^{2}, \\
c_{F A L}= & 39.624(0.005)+\frac{37}{144} \pi^{2} L_{\mu}-\frac{1}{12} \pi^{2} L_{\mu}^{2}, \\
c_{F H L}= & -\frac{557}{162}+\frac{26}{81} \pi^{2}, \\
c_{F L L}= & -\frac{163}{162}-\frac{4}{27} \pi^{2}, \\
c_{F F H}= & -0.846(0.006)-\frac{1}{20} \pi^{2} L_{\mu}, \\
c_{F A H}= & -0.098(0.051), \\
c_{F H H}= & -\frac{427}{162}+\frac{158}{2835} \pi^{2}+\frac{16}{9} \zeta(3) .
\end{aligned}
$$

All uncertainties originating from the individual master integrals are added quadratically. In order to obtain a conservative error estimate we interpret the uncertainty of the numerical integration as one standard deviation from a Gaussian distribution and multiply it by a factor of five which is accounted for in Eq. (8) [45]. The coefficients of $L_{\mu}$ could be obtained in analytic form since all renormalization constants are known analytically.

In most applications it is sufficient to know the result for the matching coefficient with numerically evaluated color factors. Setting $C_{F}=4 / 3, C_{A}=3, T=1 / 2$ and $n_{h}=1$ before inserting the master integrals and combining the numerical uncertainties we get

$$
\begin{aligned}
c_{v} \approx 1 & -2.667 \frac{\alpha_{s}^{\left(n_{l}\right)}}{\pi}+\left(\frac{\alpha_{s}^{\left(n_{l}\right)}}{\pi}\right)^{2}\left[-44.551+0.407 n_{l}\right] \\
& +\left(\frac{\alpha_{s}^{\left(n_{l}\right)}}{\pi}\right)^{3}\left[-2091(2)+120.66(0.01) n_{l}\right. \\
& \left.-0.823 n_{l}^{2}\right]+ \text { singlet terms },
\end{aligned}
$$

where $\mu=m_{Q}$ has been chosen. Note that the $n_{l^{-}}$ independent three-loop term contains the contribution with a closed massive quark loop which amounts to $\left.c_{v}^{(3)}\right|_{n_{h}} ^{n_{l}=0} \approx-0.93(8)[22]$. One observes that for $n_{l}=3,4$ and 5 all coefficients in Eq. (9) have the same sign and that they grow quite rapidly when going from NLO to NNNLO. At NNLO and NNNLO the fermionic corrections screen the non-fermionic ones, but even for $n_{l}=5$ only a reduction of at most $30 \%$ is obtained. A first glance at Eq. (9) would suggest that for the quantity $c_{v}$ perturbation theory breaks down even though the momentum scale involved in the problem, $m_{Q}$, is quite large. However, as already mentioned above, $c_{v}$ itself does not represent a physical quantity. It has to be combined with contributions originating from soft, potential and ultrasoft momentum regions which can compensate the large coefficients in Eq. (91). Further discussions on this topic can be found in Ref. 20]. It might very well be that the $\overline{\mathrm{MS}}$ scheme adopted in our calculation is not well suited 


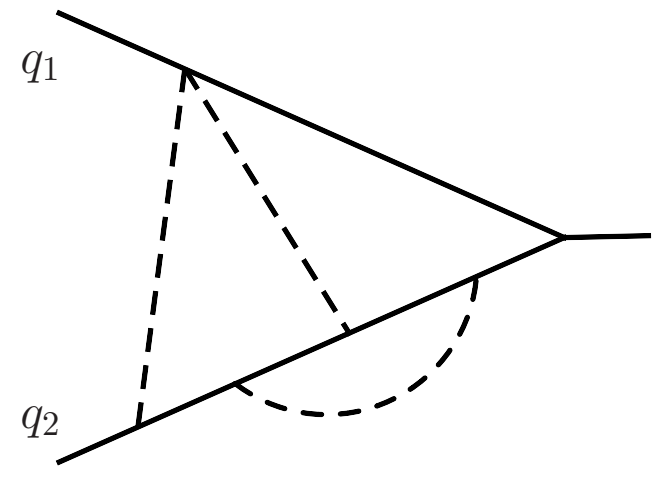

FIG. 2: Typical master integral appearing in our calculation. The solid lines and dashed lines represent massive and massless lines, respectively. For the external momenta we have the conditions $q_{1}^{2}=q_{2}^{2}=m_{Q}^{2}$ and $\left(q_{1}+q_{2}\right)^{2}=4 m_{Q}^{2}$.

for separating the divergences occuring in the different regimes. In fact, also the ultrasoft contribution studied in Ref. [11, 12] shows large numerical effects.

We have performed several checks on the correctness of our result which we want to mention in the following. In our calculation we allowed for a general gauge parameter $\xi$ which manifests as a polynomial dependence of the individual diagrams. After summing the three-loop results for $Z_{2}$ and $\Gamma_{v}$ (taking into account the corresponding quark mass counterterm contribution) we concentrated on the coefficient of the linear $\xi$-dependence and have verified that it vanishes. As a further check we recomputed the $n_{l}$ contribution [21] using our automated setup. In this context we want to mention that in Ref. 21] all occuring master integrals have been computed either analytically or using a numerical method different from the one used in the present paper. As already mentioned above, with our calculation we could also reproduce the renormalization constant in Eq. (5) with high accuracy which checks all but the highest $\epsilon$ coefficients of the master integrals. We note in passing that we have a similar accuracy for the cancellation of the spurious poles up to seventh order occuring due to our reduction procedure.

At this point it is instructive to show a result for a typical master integral contributing to $c_{v}$. For the Feynman diagram in Fig. 22, which we need up to order $\epsilon$, we obtain with the help of FIESTA 29 31]

$$
\begin{aligned}
M= & \frac{e^{3 \epsilon \gamma_{E}}}{m_{Q}^{4}}\left(\frac{\mu^{2}}{m_{Q}^{2}}\right)^{3 \epsilon}\left(+\frac{0.411236(3)}{\epsilon^{2}}+\frac{3.4860(1)}{\epsilon}\right. \\
& \left.+34.520(2)+339.68(4) \epsilon+\mathcal{O}\left(\epsilon^{2}\right)\right)
\end{aligned}
$$

A very powerful check on the correctness of our result is provided by the change of basis for the master integrals. We employ the integral tables generated during the reduction procedure in order to re-express the master integrals, which are not known analytically, through default basis (cf. Eq. (8)) alternative basis

\begin{tabular}{c|c|c}
\hline$c_{F F F}$ & $36.55(0.11)$ & $36.61(2.93)$ \\
$c_{F F A}$ & $-188.10(0.17)$ & $-188.04(2.91)$ \\
$c_{F A A}$ & $-97.81(0.08)$ & $-97.76(2.05)$ \\
$c_{v}^{(3)}\left(n_{l}=4\right)$ & $-1621.7(0.4)$ & $-1621(23)$ \\
$c_{v}^{(3)}\left(n_{l}=5\right)$ & $-1508.4(0.4)$ & $-1507(23)$
\end{tabular}

TABLE I: Comparison of the purely gluonic coefficients of Eq. (8) and $c_{v}^{(3)}$ with $n_{l}=4$ and $n_{l}=5$ for two different choices of the master integral basis. For convenience $\mu=m_{Q}$ has been adopted. The given uncertainties are obtained by combining the numerical uncertainties of each master integral contribution in quadrature. In contrast to Eqs. (8) and (9) no factor five has been introduced for this comparison.

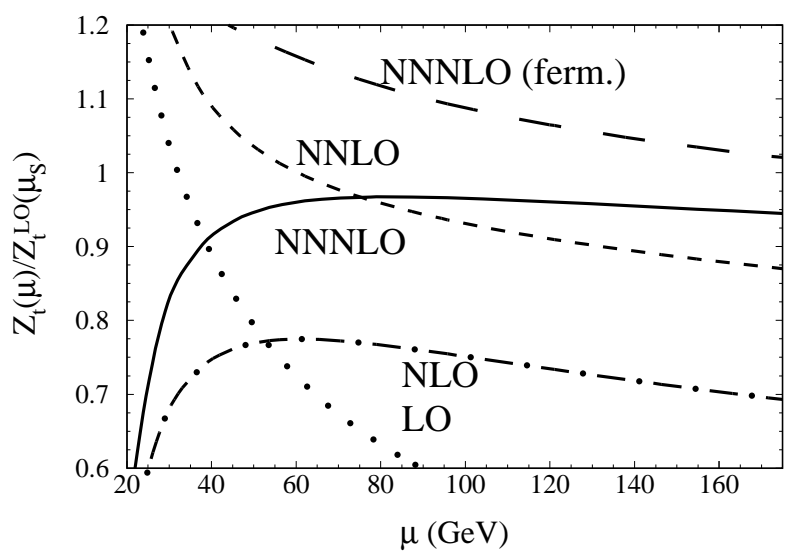

FIG. 3: Residue for the top quark system normalized to $Z_{t}^{\mathrm{LO}}\left(\mu_{S}\right)$ as a function of the renormalization scale $\mu$. Dotted, dash-dotted, short-dashed and solid lines correspond to LO, NLO, NNLO and NNNLO prediction. In the long-dashed curve only the fermion contributions to $c_{v}^{(3)}$ are taken into account (NNNLO (ferm.)).

different, in general more complicated ones. This transformation is done analytically for general space-time dimension $D$. In a next step the new master integrals are again evaluated with FIESTA and inserted in the new expression for $c_{v}$. In Tab. II we compare the results for the purely gluonic coefficients and the complete result for $c_{v}^{(3)}$ obtained in the two bases. We observe an excellent agreement within the uncertainties. In the case of the "alternative basis" one has to keep in mind that the integrals to be evaluated numerically are significantly more complicated which explains the larger uncertainties for the coefficients in Tab. [.

As a further check on the numerical evaluation of the master integrals we have used a different momentum assignment in the input for FIESTA. As a consequence different expressions are generated in intermediate steps leading to different numerical integrations. The final results are in complete agreement with Eq. (9). 
We are now in the position to have a first look to the phenomenological consequences of our result for $c_{v}$. We consider the residue of the two-point function of the vector currents

$$
\left(-q^{2} g_{\mu \nu}+q_{\mu} q_{\nu}\right) \Pi\left(q^{2}\right)=i \int \mathrm{d} x e^{i q x}\left\langle 0\left|T j_{\mu}(x) j_{\nu}^{\dagger}(0)\right| 0\right\rangle
$$

which is obtained by considering $\Pi\left(q^{2}\right)$ close to the $Q \bar{Q}$ threshold. In this limit $\Pi\left(q^{2}\right)$ is dominated by pole contributions originating from bound-state effects

$$
\Pi\left(q^{2}\right) \stackrel{E \rightarrow E_{n}}{=} \frac{N_{c}}{2 m_{Q}^{2}} \frac{Z_{n}}{E_{n}-(E+i 0)}+\ldots
$$

where the ellipsis denotes contributions from the continuum. $Z_{n}$ and $E_{n}$ are the residue and energy of the $n^{\text {th }}$ resonance which determine the height and position of the threshold cross section, respectively. In the following we consider the residue of the $1 S$ (pseudo) bound state of top quarks and extend the considerations of Ref. [16] [46] by including the non-fermionic contribution of $c_{v}^{(3)}$ and the $\mathcal{O}(\epsilon)$ term of the $1 / m_{Q}$ potential [20]. Choosing the potential subtracted scheme [40] with $\mu_{f}=20 \mathrm{GeV}$ to define the top quark mass we obtain $m_{t}^{\text {PS }}=171.4 \mathrm{GeV}$ which leads to

$$
\begin{aligned}
Z_{t}= & \frac{\left(C_{F} m_{t}^{\mathrm{PS}} \alpha_{s}\right)^{3}}{8 \pi}\left[1+(-2.131+3.661 L) \alpha_{s}+(8.38\right. \\
& \left.+1.27 x_{f}-7.26 \ln \alpha_{s}-13.40 L+8.93 L^{2}\right) \alpha_{s}^{2} \\
& +\left(5.46+\left(-2.23+0.78 L_{f}\right) x_{f}+2.21_{a_{3}}\right. \\
& +21.48_{b_{2} \epsilon}+37.53_{c_{f}}-134.8(0.1)_{c_{g}} \\
& +(-9.79-44.27 L) \ln \alpha_{s}-16.35 \ln ^{2} \alpha_{s} \\
& \left.+\left(53.17+4.66 x_{f}\right) L-48.18 L^{2}+18.17 L^{3}\right) \alpha_{s}^{3} \\
& \left.+\mathcal{O}\left(\alpha_{s}^{4}\right)\right] \\
= & \frac{\left(C_{F} m_{t}^{\mathrm{PS}} \alpha_{s}\right)^{3}}{8 \pi}\left[1-2.13 \alpha_{s}+23.66 \alpha_{s}^{2}\right. \\
& \left.-113.0(0.1) \alpha_{s}^{3}+\mathcal{O}\left(\alpha_{s}^{4}\right)\right]
\end{aligned}
$$

where $x_{f}=\mu_{f} /\left(m_{t}^{\mathrm{PS}} \alpha_{s}\right), L=\ln \left(\mu /\left(m_{t}^{\mathrm{PS}} C_{F} \alpha_{s}\right)\right)$, and $L_{f}=\ln \left(\mu^{2} / \mu_{f}^{2}\right)$. We have used $\alpha_{s}\left(M_{Z}\right)=0.1184$ to compute $\alpha_{s}=\alpha_{s}\left(\mu_{S}\right) \approx 0.141$ where the soft scale $\mu_{S}=m_{Q} C_{F} \alpha_{s}\left(\mu_{S}\right) \approx 32.16 \mathrm{GeV}$ has been adopted after the second equality sign. In order to get an impression about the importance of the individual contributions we mark the $\mu$-independent coefficients from the threeloop static potential $\left(a_{3}\right)$, from the two-loop $\mathcal{O}(\epsilon)$ term of the $1 /\left(m_{Q} r^{2}\right)$ potential $\left(b_{2} \epsilon\right)$, and from the three-loop fermion $\left(c_{f}\right)$ and purely gluonic $\left(c_{g}\right)$ contribution to $c_{v}^{(3)}$ separately. For this choice of $\mu$ one observes quite big NNNLO contributions which are dominated by $c_{g}$. Thus, it is instructive to investigate the $\mu$-dependence of $Z_{t}$ which is shown in Fig. 3. Around the soft scale no convergence is observed. Allowing, however, for higher scales one finds a quite flat behavior of the NNNLO curve. Furthermore, the NNNLO corrections become quite small. E.g., considering the top quark system for $\mu \approx 80 \mathrm{GeV}$, the NLO terms amount to about $+15 \%$ and the NNLO to roughly $+20 \%$. The third-order contribution is practically zero. Similar observations also hold for the bottom quark case, see Ref. [20].

\section{CONCLUSIONS}

The third-order contribution to the matching coefficient of the vector current between QCD and NRQCD has been computed. An automated setup has been developed where even the occuring master integrals are identified automatically, processed with the help of the computer program FIESTA, and prepared for the insertion into the analytic reduction of $c_{v}^{(3)}$.

In the $\overline{\mathrm{MS}}$ scheme the numerical impact of $c_{v}^{(3)}$ is quite big as can be seen from Eq. (9) which constitutes the main result of this paper. In a dedicated analysis one has to investigate the consequences for the bottom-quark mass extracted from $\Upsilon$ sum rules and the top-quark threshold production cross section at a future linear collider.

An analysis of the residue of the $1 S$ state indicates that at energy scales around two to three times the soft scale good convergence of the perturbative series is observed.

\section{Acknowledgments}

We would like to thank Martin Beneke and Alexander Penin for many useful discussions and communications and Alexander Smirnov for continuous support on FIESTA. This work is supported by DFG through SFB/TR 9 "Computational Particle Physics". The Feynman diagrams were drawn with the help of Axodraw [41] and JaxoDraw [42].
[1] W. E. Caswell and G. P. Lepage, Phys. Lett. B 167 (1986) 437.

[2] G. T. Bodwin, E. Braaten and G. P. Lepage, Phys. Rev. D 51 (1995) 1125 [Erratum-ibid. D 55 (1997) 5853] arXiv:hep-ph/9407339.
[3] N. Brambilla et al., arXiv:hep-ph/0412158

[4] A. Pineda and A. Signer, Phys. Rev. D 73 (2006) 111501 arXiv:hep-ph/0601185.

[5] A. Hoang, P. Ruiz-Femenia and M. Stahlhofen, JHEP 1210 (2012) 188 [arXiv:1209.0450 [hep-ph]]. 
[6] A. H. Hoang et al., Eur. Phys. J. directC 2 (2000) 1 arXiv:hep-ph/0001286.

[7] M. Martinez and R. Miquel, Eur. Phys. J. C 27 (2003) 49 arXiv:hep-ph/0207315.

[8] K. Seidel, F. Simon, M. Tesar and S. Poss, Eur. Phys. J. C 73 (2013) 2530 arXiv:1303.3758 [hep-ex]].

[9] T. Horiguchi, A. Ishikawa, T. Suehara, K. Fujii, Y. Sumino, Y. Kiyo and H. Yamamoto, arXiv:1310.0563 [hepex].

[10] J. Beringer et al. [Particle Data Group Collaboration], Phys. Rev. D 86 (2012) 010001.

[11] M. Beneke, Y. Kiyo and A. A. Penin, Phys. Lett. B 653 (2007) 53 arXiv:0706.2733 [hep-ph]].

[12] M. Beneke and Y. Kiyo, Phys. Lett. B 668 (2008) 143 arXiv:0804.4004 [hep-ph]].

[13] A. V. Smirnov, V. A. Smirnov and M. Steinhauser, Phys. Lett. B 668 (2008) 293 arXiv:0809.1927 [hep-ph]].

[14] A. V. Smirnov, V. A. Smirnov and M. Steinhauser, Phys. Rev. Lett. 104 (2010) 112002 arXiv:0911.4742 [hep-ph]].

[15] C. Anzai, Y. Kiyo and Y. Sumino, Phys. Rev. Lett. 104 (2010) 112003 arXiv:0911.4335 [hep-ph]].

[16] M. Beneke, Y. Kiyo, A. Penin and K. Schuller, eConf C 0705302 (2007) TOP01 arXiv:0710.4236 [hep-ph]].

[17] M. Beneke, Y. Kiyo and K. Schuller, PoS RADCOR 2007 (2007) 051 [arXiv:0801.3464 [hep-ph]].

[18] M. Beneke, Y. Kiyo and K. Schuller, arXiv:1312.4791 [hep-ph].

[19] M. Beneke, Y. Kiyo and K. Schuller, to be published.

[20] M. Beneke, Y. Kiyo, P. Marquard, A. Penin, J. Piclum, D. Seidel and M. Steinhauser, to be published.

[21] P. Marquard, J. H. Piclum, D. Seidel and M. Steinhauser, Nucl. Phys. B 758 (2006) 144 arXiv:hep-ph/0607168.

[22] P. Marquard, J. H. Piclum, D. Seidel and M. Steinhauser, Phys. Lett. B 678 (2009) 269 [arXiv:0904.0920 [hep-ph]].

[23] D. J. Broadhurst, N. Gray and K. Schilcher, Z. Phys. C 52 (1991) 111.

[24] K. Melnikov and T. van Ritbergen, Nucl. Phys. B 591 (2000) 515 arXiv:hep-ph/0005131.

[25] P. Marquard, L. Mihaila, J. H. Piclum and M. Steinhauser, Nucl. Phys. B $\mathbf{7 7 3}$ (2007) 1 arXiv:hep-ph/0702185.

[26] M. Beneke and V. A. Smirnov, Nucl. Phys. B 522 (1998) 321 arXiv:hep-ph/9711391.

[27] V. A. Smirnov, Analytic tools for Feynman integrals, Springer Tracts Mod. Phys. 250 (2012) 1.
[28] P. Marquard and D. Seidel, unpublished.

[29] A. V. Smirnov and M. N. Tentyukov, Comput. Phys. Commun. 180 (2009) 735 arXiv:0807.4129 [hep-ph].

[30] A. V. Smirnov, V. A. Smirnov and M. Tentyukov, Comput. Phys. Commun. 182 (2011) 790 arXiv:0912.0158 [hep-ph]].

[31] A. V. Smirnov, arXiv:1312.3186 [hep-ph].

[32] M. Beneke, A. Signer and V. A. Smirnov, Phys. Rev. Lett. 80 (1998) 2535 arXiv:hep-ph/9712302.

[33] B. A. Kniehl, A. A. Penin, M. Steinhauser and V. A. Smirnov, Phys. Rev. Lett. 90 (2003) $212001 \quad$ [Erratum-ibid. $91 \quad$ (2003) $139903(\mathrm{E})]$ arXiv:hep-ph/0210161.

[34] B. A. Kniehl, A. Onishchenko, J. H. Piclum and M. Steinhauser, Phys. Lett. B 638 (2006) 209 arXiv:hep-ph/0604072].

[35] G. Källen and A. Sarby, K. Dan. Vidensk. Selsk. Mat.Fis. Medd. 29, N17 (1955) 1.

[36] A. Czarnecki and K. Melnikov, Phys. Rev. Lett. 80 (1998) 2531 arXiv:hep-ph/9712222.

[37] E. Braaten and Y. -Q. Chen, Phys. Rev. D 57 (1998) 4236 [Erratum-ibid. D 59 (1999) 079901] hep-ph/9710357].

[38] Y. Kiyo, M. Steinhauser and N. Zerf, Phys. Rev. D 80 (2009) 075005 arXiv:0907.1146 [hep-ph]].

[39] https://www.ttp.kit.edu/Progdata/ttp14/ttp14-002/.

[40] M. Beneke, Phys. Lett. B 434 (1998) 115 hep-ph/9804241.

[41] J. A. M. Vermaseren, Comput. Phys. Commun. 83 (1994) 45.

[42] D. Binosi and L. Theussl, Comput. Phys. Commun. 161 (2004) 76 arXiv:hep-ph/0309015.

[43] Due to Furry's theorem the two-loop singlet contribution is zero for the vector current.

[44] Note that in Ref. [21] the result has been expressed in terms of the coupling defined in the full theory whereas here we use the effective one denoted by $\alpha_{s}^{\left(n_{l}\right)}$. This explains the difference in the logarithmic part of the coefficient $c_{F H L}$.

[45] Note that in Ref. [22], where the fermionic contributions are given, only a factor two has been chosen which explains the slight increase of the uncertainty of $c_{F A H}$ in Eq. (8).

[46] Note that there is a misprint in Eq. (5) of Ref. [16]: the term $E\left(1-d_{v} / 3\right) / m$ should read $E\left(c_{v}-d_{v} / 3\right) / m$. 